\title{
Ich und die anderen - Freiheit und/versus Schutz
}

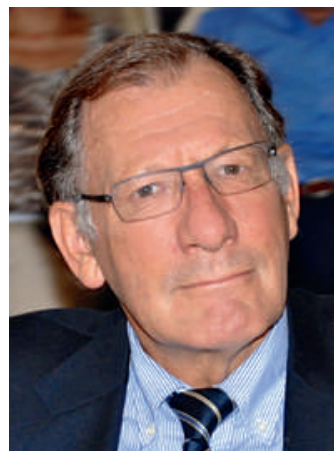

Jean Martin
Kürzlich sprach ich [1] von vielfältigen Wahrheiten in unterschiedlichen Gesellschaften. Bei einem Besuch in den USA gab mir ein Freund ein Buch, in dem sozialethische Positionen vertreten sind, die die «Extremisten der Mitte» (denen ich angehöre) bestürzen. Das Buch thematisierte die Entwicklung der Rassenbeziehungen, darunter insbesondere die Sklaverei, und hinterfragte die Bedeutung von Freiheit [2]. Unter anderem beschrieb der Autor eine schrittweise Verhärtung der Beziehung zwischen Schwarz und Weiss - zum Nachteil der schwarzen Bevölkerung während im wenig kodifizierten Kontext des 16. Jahrhunderts eine gewisse Integration beider Gruppen möglich war.

Zur Freiheit schreibt Richter Napolitano: «Die Frage der nationalen Sicherheit [der Text macht deutlich, dass der Autor seine Position auf verschiedene Formen von Sicherheit/Schutz anwendet, darunter auch auf die soziale Sicherheit] liefert einen Bezugspunkt für die Beurteilung der Frage, inwieweit die natürliche Freiheit (basierend auf den snatürlichen Rechten`) über dem jeweiligen Handlungswillen einer Regierung steht, vor allem heute, wo viel die Rede vom neu zu findenden Gleichgewicht zwischen Freiheit und Sicherheit ist. Meine Erfahrungen als Mitglied des Obersten Gerichtshofes von New Jersey und Beobachter der Gesellschaft bringen mich zu der Überzeugung, dass die Beziehung zwischen Freiheit und Sicherheit in unserem Lande keine Frage des Gleichgewichts zwischen den beiden Begriffen ist, sondern dass das Pendel immer in Richtung Freiheit ausschlägt. Regieren steht an sich schon für das Negieren von Freiheit. Es entspricht dem ureigensten Wesen von Regierungen, den Menschen ihre natürlichen Rechte zu entziehen. Sicherheit ist ein Konstrukt. Alles, was eine Regierung tut, muss zunächst einmal als verdächtig angesehen werden. Während [die Umsetzung jeglicher Massnahmen zur] Sicherheit einer entsprechend adäquaten Rechtfertigung bedarf, ist dies bei der Ausübung individueller natürlicher Rechte nie der Fall.»

In der Behauptung, dass die Ausübung von Freiheit keiner Rechtfertigung bedarf, unterscheiden wir uns (deutlich!): In der Tat darf der andere nicht ungerechtfertigt verletzt werden («die Freiheit des einen endet da, wo die Freiheit des anderen anfängt»). Man fragt sich, inwieweit A. P. Napolitano bedenkt, dass nicht alle Menschen in der Lage sind, ihre legitimen Interessen zu verteidigen (ungleich sind). Angesichts solcher Aussagen überrascht nicht weiter, dass beispielsweise das im Laufe der Jahre von Frankreich entwickelte soziale System von manchen in den USA als kommunistisch angesehen wird. Hier wird eine deutliche Kluft aufgezeigt zwischen Ländern, die doch im Wesentlichen mit einem gemeinsamen ethischen Rüstzeug ausgestattet sind, das ein Autor als Erbe «griechischer Philosophen, römischer Gesetzgeber und jüdischer Propheten» bezeichnet hat.

Die Ablehnung aller staatlichen Einschränkungen lässt die Errungenschaften einer Gesellschaft ausser Acht, die davon ausgeht, dass Gesetze erforderlich sind, um Benachteiligte zu schützen. Dazu Lacordaire: «Zwischen den Starken und den Schwachen, zwischen Herren und Knechten sorgt die Freiheit für Unterdrückung und das Gesetz für Freiheit.» Uns näher, mit Blick auf den Rassismus, sagte Martin Luther King: «Alle Gesetze, die den Menschen erhöhen, sind gerecht. Alle, die ihn erniedrigen, sind ungerecht.»

Einer unserer Lehrer der öffentlichen Gesundheit meinte, dass es in der Politik - einfach ausgedrückt - um die Entscheidung ginge, «wer erhält was?», d.h. um das ständige Spannungsverhältnis zwischen Freiheit und Willen/Verantwortung für solidarisches Verhalten. Bei dieser Gelegenheit ist anzumerken, dass politisch liberale Gesellschaften Präventivmassnahmen kaum begünstigen. Sie gelten als freiheitsberaubend. Solche Gesellschaften handeln oder sanktionieren in der Regel erst, wenn der Schaden auftritt. Dies gilt für Gesundheitsprävention und Grenzen, die externen Risiken zu setzen sind.; besonders, heutiges Beispiel, für die amerikanische Debatte über die Kontrolle von Waffen, bei der Extrempositionen in Bezug auf die persönliche Freiheit verblüffen. Libertäre sind zwar nicht naiv, doch scheinen sie zu glauben, dass jede regulatorische Handlung des Staates die grundsätzlich gute Natur des Menschen nur schädigen kann. Rousseau-Schüler? Ich bin nicht sicher, ob Jean-Jacques erfreut wäre über solche Schüler [3].

Die Systemwissenschaft macht deutlich, dass komplexe Systeme stabiler sind. Extrem doktrinäre Positionen übersehen die Anfälligkeit von Systemen, die sich (fast) nur auf einen einzigen Pfeiler stützen: bei Napolitano die Sakralisierung der unantastbaren Freiheit, anderswo die Überzeugung, dass Reglementierung und eine starke Hand des Staates wichtigster Garant sind. Früher oder später zeigt die Geschichte, dass die Anfälligkeit «eingleisiger» Systeme deren jeweilige Führung zu autoritärem - und freiheitsberaubendem - Handeln zwingt. Gefragt ist und bleibt die richtige Mitte.

Jean Martin, Mitglied der Redaktion und der Nationalen Ethikkommission 